\title{
Examination of Far-Field Mathematical Absorber Reflection Suppression through Computational Electromagnetic Simulation
}

\author{
S. F. Gregson, A. C. Newell, and G. E. Hindman \\ Nearfield Systems Inc., 19730 Magellan Drive, Torrance, CA 90502, USA \\ Correspondence should be addressed to S. F. Gregson, sgregson@nearfield.com
}

Received 13 October 2011; Accepted 11 March 2012

Academic Editor: Lars Jacob Foged

Copyright () 2012 S. F. Gregson et al. This is an open access article distributed under the Creative Commons Attribution License, which permits unrestricted use, distribution, and reproduction in any medium, provided the original work is properly cited.

The mathematical absorber reflection suppression (MARS) technique has been used to identify and then suppress the effects of spurious scattering within spherical, cylindrical, and planar near-field antenna measurement systems, compact antenna test ranges (CATRs), and far-field measurement facilities for some time now. The recent development of a general-purpose threedimensional computational electromagnetic model of a spherical antenna test system has enabled the MARS measurement and postprocessing technique to be further investigated. This paper provides an overview of the far-field MARS technique and presents an introduction to the computational electromagnetic range model. Preliminary results of computational electromagnetic range simulations that replicate typical MARS measurement configurations are presented and discussed which, for the first time, confirm through simulation many of the observations that have previously been noted using purely empirical techniques.

\section{Introduction to Far-Field MARS}

Reflections in antenna test ranges can often be the largest source of measurement error within the error budget of a given facility [1] with direct collimating ranges being perhaps the most susceptible to these contaminants [2]. Considerable attention has been paid to range multipath suppression in the open literature with significant effort, ingenuity and resourcefulness having been devoted to quantifying and subsequently correcting multipath contaminated measurements by means of hardware or software time gating, background subtraction, complex plane circular least squares fitting, and signal-encoding-based techniques. However, until very recently, the frequency domain mode orthogonalisation and filtering techniques that have proved so overwhelmingly successful in near-field measurements (i.e., spherical [36], cylindrical [7-9], planar [10-12], and more generally [13]) have not been applied to one-dimensional farfield measurements. Far-field MARS (F-MARS) $[14,15]$ is very closely related to the well-established spherical and cylindrical MARS implementations with processed results being obtained from only a single one-dimensional far-field pattern cut. This is significant, as one of the most appealing attributes of the far-field methodology has been its ability to provide a single antenna pattern cut, thereby, minimising the required measurement time and complexity. However, one of the more widely acknowledged shortcomings of making direct far-field antenna pattern measurements is that range multipath can degrade the accuracy of the measurement results [2]. This is especially true for outdoor far-field measurements where, in general, far less control is obtained over the test environment. The F-MARS measurement and post-processing technique was specifically conceived for use with far-field ranges to combat exactly this issue where only one-dimensional single frequency far-field antenna pattern data is acquired.

F-MARS is entirely generic in nature, and can be applied to a variety of different antenna types with no a priori assumptions being made about the excitation or distribution of currents sources. Previously [14, 15], empirical test campaigns have been used to verify that the F-MARS technique is able to suppress spurious range reflections whilst preserving the integrity of the underlying antenna pattern function. This verification has been accomplished by establishing the degree of repeatability between successive F-MARS processed measurements where only a single parametric change had been introduced into the experimental configuration. That parametric change comprised the installation of a single 
large scattering object into the test environment. During that investigation, it was found that like other implementations of the MARS technique, displacing the antenna under test (AUT) away from the centre of rotation was crucial to the success of the technique, and rules for optimising this offset were developed. The purpose of this paper is to present the results of a computational electromagnetic simulation that attempted to recreate the previously used experimental configuration and procedure in order to obtain further independent verification of the F-MARS technique. Whilst a detailed description of the theoretical basis and practical verification of F-MARS can be found presented in the open literature $[14,15]$ and is not the primary subject of this paper, the following summary of the F-MARS measurement and data postprocessing may be of some utility to the reader in understanding the chosen simulation configuration described herein.

(1) Take a direct acquisition of the one-dimensional far electric field amplitude and phase pattern function with the AUT offset from the origin (a single far-field component is sufficient).

(2) Apply a differential phase change to mathematically translate the AUT to the origin of the measurement coordinate system.

(3) Obtain the translated mode coefficients of the AUT for an AUT conceptually located at the origin of the measurement coordinate system using the inverse fast Fourier transform (FFT).

(4) Apply band-pass mode filtering function to suppress unwanted higher order cylindrical mode coefficients (CMC) where the properties of the filter function are determined from the physical size of the AUT and the free space propagation number.

(5) Compute the complete far electric field pattern from the filtered mode coefficients using the FFT to obtain the MARS filtered antenna pattern function.

Here, since these transformations and their inverse operations can be evaluated using the one-dimensional fast Fourier transform (FFT) algorithm, this insures that FMARS processing is very efficient in terms of computational effort and resources.

The development of general-purpose tools for the simulation of near-field or far-field antenna measurements is of interest for several reasons. Such a tool would enable one to plan and optimise a measurement campaign before committing valuable facility time or resources or to assess individual error terms within the facility level error budget and to verify correction algorithms. In this instance it was the impact of spurious range multipath that was being assessed together with the effectiveness of correction techniques. In general, it is difficult to obtain closed form functional solutions for the electromagnetic (EM) field at an arbitrary point in space from knowledge of the tangential electric and/or tangential magnetic fields over a closed surface for anything but the simplest of configurations. This is especially true when the closed surface is not coincident with the aperture of the radiating structure, as is the case for near-field or MARS type antenna measurements. As such, recourse to alternative, typically numerical-based techniques becomes unavoidable.

In essence, any antenna measurement can be simulated by evaluating the complex coupling coefficient between the AUT and the field probe (or remote source antenna). This must be accomplished for each point within the simulated acquisition surface, for each sampled polarisation, and for each frequency at which the measurement is to be taken. In principle then, it would be possible to obtain the mutual coupling coefficient, $S_{21}$, between a given mode in the waveguide port in the AUT and a given mode in the waveguide port on the scanning probe (or remote source antenna) from a three-dimensional computational electromagnetic (CEM) full wave solver. This approach would have the advantage of, potentially, introducing the least number of assumptions and approximations and therefore could in principle yield the most accurate predictions. Unfortunately, at the present time, although many solvers are available employing, say, the finite difference time domain (FDTD) method, the finite element method (FEM), the method of moments (MoM), and so forth, these are generally considered inappropriate for simulating problem spaces as electrically large as those needed to enclose a complete near- or far-field measurement system, especially when that model extends to include positioners, absorber, cranes, and lights. This limitation is merely a consequence of the extended processing times and the large amounts of computer resources that are typically required. Hence, alternative, perhaps less generally applicable, but more computationally efficient techniques are required. One possible strategy for accomplishing this is introduced in the following section.

\section{Overview of Simulation Technique}

The computational electromagnetic simulation of a direct far-field range measurement was based on physical optics, and specifically on the Kirchhoff-Huygens principle which enables fields specified over one closed surface to be propagated to another point in space. The Kirchhoff-Huygens principle is a powerful technique for determining the field in a source free region outside a closed surface from the knowledge of the electromagnetic field distributed across that surface. This method is applicable to arbitrary shaped surfaces over which both the electric and magnetic fields are known. The form of the Huygens principle which is used can be derived from the integral form of the Stratton Chu equations [16] which represents an integral form of Maxwell's equations. When expressed mathematically, the electric field at a point $P$ radiated by a closed, but arbitrary, Huygens surface $S$ is

$$
\begin{aligned}
\underline{E}_{p}=\frac{1}{4 \pi} \int_{S}- & {[j \omega \mu(\underline{n} \times \underline{H}) \psi+(\underline{n} \times \underline{E})} \\
& \left.\times \nabla_{0} \psi+(\underline{n} \cdot \underline{E}) \nabla_{0} \psi\right] d s_{0} .
\end{aligned}
$$


Here, $\underline{E}$ and $\underline{H}$ are the electric and magnetic fields, respectively, and are specified over the enclosing surface, and $\psi$ denotes the first order spherical function,

$$
\psi=\frac{e^{-j k_{0} r^{\prime}}}{r^{\prime}} .
$$

Here $r^{\prime}$ is the displacement of the field point from the elemental source and is related to the coordinates of the elemental Huygens source $r_{0}$ and the coordinates of the field point $r$ through,

$$
r^{\prime}=\left|\underline{r}-\underline{r_{0}}\right| .
$$

$\omega$ is the angular frequency and is related to the frequency $f$ by $\omega=2 \pi f ; k_{0}$ is the free space propagation constant and is related to the wavelength by $k_{0}=2 \pi / \lambda ; \underline{n}$ is the outward pointing unit normal and, respectively; $\varepsilon$ and $\mu$ are the permittivity and permeability of the medium through which the fields are propagating. In general, these are complex tensors that are a function of the field strength; however, here it is assumed that the region of space under consideration is free space comprising a homogeneous isotropic linear dielectric medium, and thus $\varepsilon$ and $\mu$ can be approximated by real constants. $j$ is the imaginary unit, and $\nabla_{0}$ is the differential vector operator expressed in the source coordinate system. The geometry of this statement of the Kirchhoff-Huygens formula can be found illustrated in its conventional form in Figure 1.

Expanding $\nabla_{0} \psi$ obtains [17]

$$
\nabla_{0} \psi=\left(j k_{0}+\frac{1}{r^{\prime}}\right) \underline{\hat{r}^{\prime}} \psi
$$

Thus, the general vector Kirchhoff-Huygens formula can be expressed as [15]

$$
\begin{aligned}
\underline{E}_{p}=\frac{1}{4 \pi} \int_{S}- & {\left[j \omega \mu(\underline{n} \times \underline{H})+\left\{(\underline{n} \times \underline{E}) \times \underline{\hat{r}^{\prime}}+(\underline{n} \cdot \underline{E}) \underline{\underline{r}}\right\}\right.} \\
& \left.\times\left(j k_{0}+\frac{1}{r^{\prime}}\right)\right] \times \frac{e^{-j k_{0} r^{\prime}}}{r^{\prime}} d s .
\end{aligned}
$$

These expressions yield the vector electric field function from an integral of the electric and magnetic fields over the closed surface $S$ where $d S$ is an elemental portion of the that surface. Equivalent expressions can be obtained for the magnetic field [16-18]. These expressions are amenable for evaluating the field at any point in space outside of $S$ and as such can be used to create near- and far-field simulations alike. The use of these expressions enables commercially available full-wave three-dimensional CEM simulation tools to be used to solve the fields around some, comparatively small tractable, radiating structure whereupon the Kirchhoff-Huygens method can be used to calculate the fields resulting from this radiator throughout a much larger problem space. In this way, measurement simulations of great accuracy can be produced comparatively simply and easily using essentially rigorous, but computationally intensive, near-field solvers.

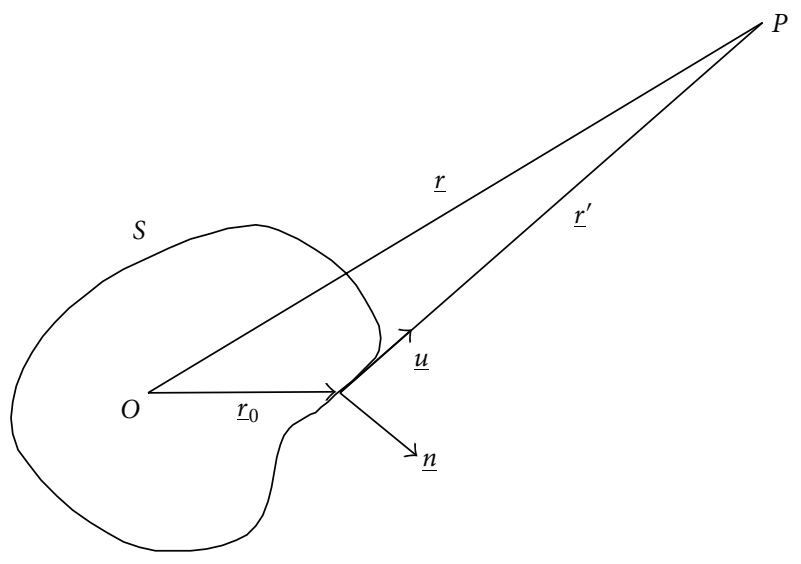

FIGURE 1: Geometry of Kirchhoff-Huygens formula, that is, (5), for the propagation of electromagnetic fields from Huygens surface $S$ to point $P$.

Typically, when using (5) to compute the far-field pattern of an antenna from knowledge of the corresponding nearfields, the vector $\underline{r}$ would be chosen so that the evaluation of the integral would produce the antenna diagram for a specified angular direction, as for example,

$$
\begin{aligned}
\underline{\underline{\hat{r}}}= & \sin (\mathrm{Az}) \cos (\mathrm{El}) \underline{\hat{e}}_{x}+\sin (\mathrm{El}) \underline{\hat{e}}_{y} \\
& +\cos (\mathrm{Az}) \cos (\mathrm{El}) \underline{\hat{e}}_{z} .
\end{aligned}
$$

Here, $\mathrm{Az}$ and $\mathrm{El}$ are the azimuth and elevation angles, respectively, and describe a conventional azimuth over elevation spherical positioning system $[17,19]$. However, when simulating an antenna measurement facility, it is perhaps simpler to rotate the AUT, that is, the closed Huygens surface, using an isometric rotation and then to evaluate the far fields in a single direction, which more closely mimics the antenna measurement process and allows chamber scatterer to be introduced into the model in a direct way. In this case, $\underline{r}$ would be held fixed for all measurement angles such that

$$
\underline{\hat{r}}=1 \underline{\hat{e}}_{z} .
$$

Such isometric rotations are easily implemented using transformation matrices to rotate the fields and coordinates. Transformation matrices are matrices that post-multiply a column point vector to produce a new column point vector. A series of transformation matrices may be concatenated into a single matrix using matrix multiplication. A transformation matrix may represent each of the operations of translation, scaling, and rotation. However, if $A$ is a three by three orthogonal, normalised square matrix, it may be used to specify an isometric rotation that can be used to relate two frames of reference, that is, two coordinate systems. Here, an isometric rotation is taken to mean a transformation in which the distance between any two points on an object remain invariant under the transformation. Any number of angular definitions for describing the relationship between the two coordinate systems exists. However, if the angles azimuth and elevation are used, where the rotations are applied in this order, we may write that a point in one frame 
of reference can be specified in terms of a point in the other frame of reference as $[17,19]$

$$
\left[\begin{array}{c}
u^{\prime} \\
v^{\prime} \\
w^{\prime}
\end{array}\right]=[A] \cdot\left[\begin{array}{l}
u \\
v \\
w
\end{array}\right] .
$$

Here primed coordinates are used to denote the rotated frame of reference. In this case, in order that a far-field system comprising an azimuth over elevation positioning system is utilised, then $A$ can be constructed by applying a rotation of elevation about the positive $x$-axis and a negative azimuth rotation about the positive $y$-axis where the rotations are applied in this order [17, 19]. Thus, by fixing $\underline{\hat{r}}=1 \hat{\hat{e}}_{z}$ and using (8) to rotate the electric and magnetic fields, the position vector $r_{0}$, the unit surface normal, and the AUT can be positioned within the range as though they were installed on a conventional azimuth over elevation positioning system. As a central constituent of the MARS measurement process is to offset the AUT from the centre of rotation, this can be easily incorporated within the simulation by applying an offset to the Cartesian components of the position vector that determines the location of the radiating Huygens surface. This translation is applied prior to applying the aforementioned rotation. Once evaluated using the Kirchhoff-Huygens method, the resulting far-fields can be resolved from the range coordinate system back onto the antenna coordinate system by applying the inverse rotation to the far electric (and magnetic) fields where the results have been found to be in agreement with those produced using conventional processing. Crucially, when introducing a given scatterer into the range simulation, the position and orientation of the scatterer is by definition specified in the range coordinate system and will therefore be fixed, irrespective of the particular far-field antenna pattern angle being computed. Thus, working in terms of the range coordinate system as outlined above significantly simplifies the computational processing.

The inclusion of an arbitrarily located and shaped perfectly conducting (i.e., worst case) scattering object can be introduced by using the Kirchhoff-Huygens field propagation method described above together with the generalised law of reflection. By definition, an elemental Huygens source is considered to be infinitesimally small, and so it will radiate a spherical wave. However, as the observation point on the reflecting plate is finitely far removed from the source, that is, more than a few wavelengths away, the reflecting plate will be in the far field of the elemental Huygens source. Locally therefore, at the observation point, the field will be of the form of a TEM plane wave propagating in the direction $\underline{r}^{\prime}$. As the field is a local plane wave and assuming that the reflecting surface is locally, planar and is made from a perfectly conducting (PEC) material, the normal electric field component will be unchanged upon reflection. Thus, if a homogeneous plane wave is incident on a perfect electrical conducting (PEC) flat surface of infinite extent, the reflected elemental electric field constitutes a similar plane wave and the reflected field can be obtained from the incident field using [18]

$$
\underline{E}_{r}=2\left(\underline{\hat{n}} \cdot \underline{E}_{i}\right) \underline{\hat{n}}-\underline{E}_{i} .
$$

The law of reflection states that the angle of incidence equals the angle of reflection. Thus, when expressed mathematically this becomes,

$$
\theta=\arccos \left(\underline{\hat{\underline{\hat{n}}}} \cdot \underline{\hat{\underline{u}}}_{i}\right)=\arccos \left(\underline{\hat{\underline{\hat{n}}}} \cdot \underline{\hat{\underline{u}}}_{r}\right) .
$$

Thus it is possible to write the general statement of reflection as [18],

$$
\underline{\hat{\underline{u}}}_{r}=\underline{\hat{\underline{u}}}_{i}-2\left(\underline{\hat{\underline{n}}} \cdot \underline{\hat{\underline{u}}}_{i}\right) \underline{\hat{\underline{n}}} .
$$

Here, $\hat{\underline{u}}_{i}$ denotes the direction of propagation of the incident plane wave, and $\hat{\underline{u}}_{r}$ represents the direction of propagation of the reflected, specular plane wave. This can be taken to represent the general form of the law of reflection with the scattered field being a plane wave as the material is assumed to be infinite in extent in the tangential direction, and the material properties do not vary across this surface. As the reflected elemental electric field correspond to a plane wave propagating in the direction $\hat{\underline{u}}_{r}$, the elemental magnetic field can be obtained from the elemental electric field using the TEM, that is, plane wave condition thus $[17,18]$

$$
d \underline{H}_{r}=\frac{1}{Z_{0}} \underline{\hat{\underline{u}}}_{r} \times \underline{E}_{r} d s .
$$

Here, $Z_{0}$ is the plane wave impedance, or characteristic impedance, of free space where $Z_{0}=c \mu_{0}$. The total reflected electric and magnetic fields at each point on the surface of the reflector can be obtained by summing all of the contributions from the many elemental Huygens sources infinitely radiated from $S$. The far scattered field can again be obtained using the Kirchhoff-Huygens method by integrating over the surface of the reflector and thereby evaluating the far scattered fields. The total measured far field for a particular pattern angle can be obtained by taking the linear superposition of the direct and scattered fields where, in agreement with theory, the remote source antenna is assumed to be an infinitesimal Hertzian dipole. As a quasifar-field measurement is being simulated, probe pattern effects are unimportant in this analysis providing that the range length is sufficiently large to insure that the pattern of the far-field range illuminator is essentially constant across the AUT during the measurement [20]. Thus, by following this process, a simulation of a typical MARS type far-field measurement can be created with almost complete freedom to choose the AUT, the measurement geometry, and the location of the scattering object in order to test the existing far-field MARS technique. Far-field predictions obtained from this simulation technique together with results of the far-field MARS processing can be found presented within the following sections.

\section{Results}

In order that the F-MARS measurement and postprocessing technique could be further verified, a far-field measurement 


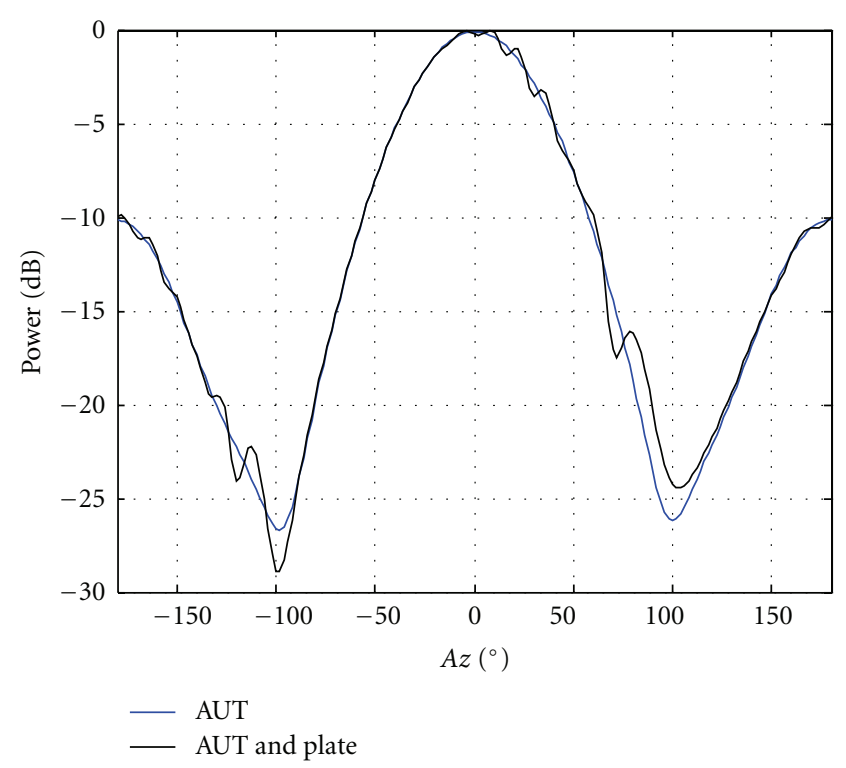

FIGURe 2: Far-field amplitude pattern of OEWG with and without plate. AUT offset $=10 \mathrm{~cm}$.

was simulated that recreated a typical F-MARS configuration. A commercially available three-dimensional full-wave CEM solver was used to simulate the near fields radiated by a WR90 open-ended rectangular waveguide (OEWG) section that was excited by a $\mathrm{TE}_{10}$ mode. The Cartesian components of the electric and magnetic fields were obtained at $10 \mathrm{GHz}$ and were specified over the surface of an ellipsoid that tightly bounded the radiating aperture. The maximum radial extent of this ellipsoid when displaced from the origin was $0.08 \mathrm{~m}$. The aperture of the OEWG section was displaced from the origin of the range measurement coordinate system, that is, the centre of rotation, by $0.1 \mathrm{~m}$ in the AUT $z$-axis, which was specified as being at a normal to the waveguide aperture plane. A square reflecting plate of side length $0.3 \mathrm{~m}$ was introduced into the simulation located with its centre at $x=$ $-0.1 \mathrm{~m}, y=0 \mathrm{~m}$, and $z=1.0 \mathrm{~m}$ with its unit normal directed in the positive $x$-axis and the sides of the plate being parallel with the $y$ - and $z$-axes. This configuration closely mimicked the experimental arrangement that had previously been used to verify the F-MARS technique, compare $[14,15]$. The physical-optics-based simulation was then used to obtain the far-electric field great circle azimuth cut with a range length of $200 \mathrm{~m}$ which placed the AUT in the far field for all of the simulations. The results of this simulation can be found presented in Figure 2, which contains the amplitude antenna diagram plotted together with the equivalent ideal far-field pattern (i.e., the far-field pattern of antenna in the absence of the reflecting flat plate). Figure 3 contains an equivalent plot that shows the far-field great circle phase pattern.

Typically, an antenna is installed within a near, or farfield facility such that it is displaced in space as little as possible during the course of a measurement. As range multipath tends to disturb the fields illuminating the test antenna, the purpose of this strategy is to ensure that the field illuminating the test antenna changes as little as possible during the course of the acquisition, thereby minimising the impact of

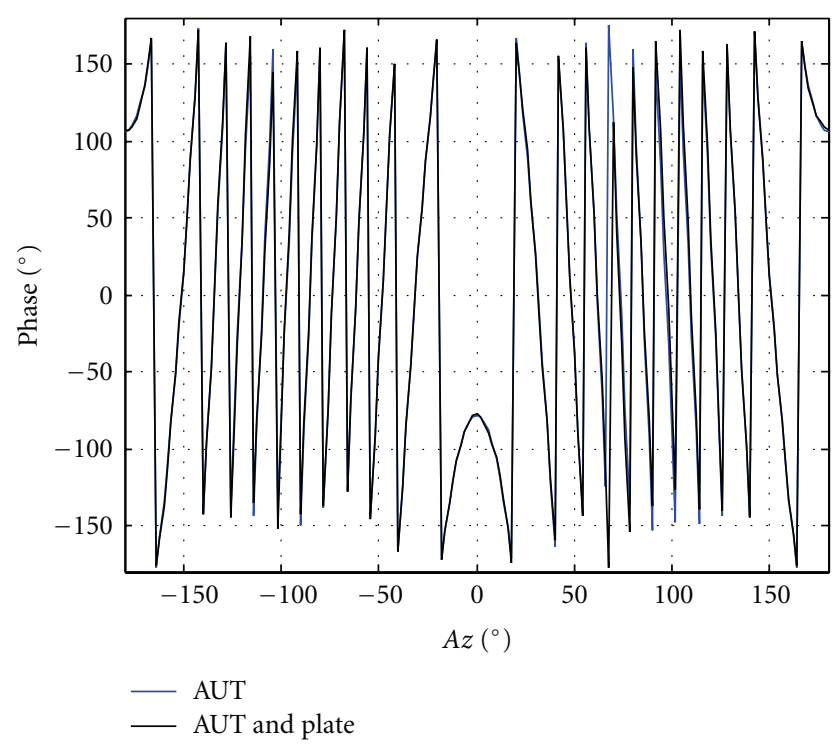

FIGURE 3: Far-field phase pattern of OEWG with and without plate. AUT offset $=10 \mathrm{~cm}$.

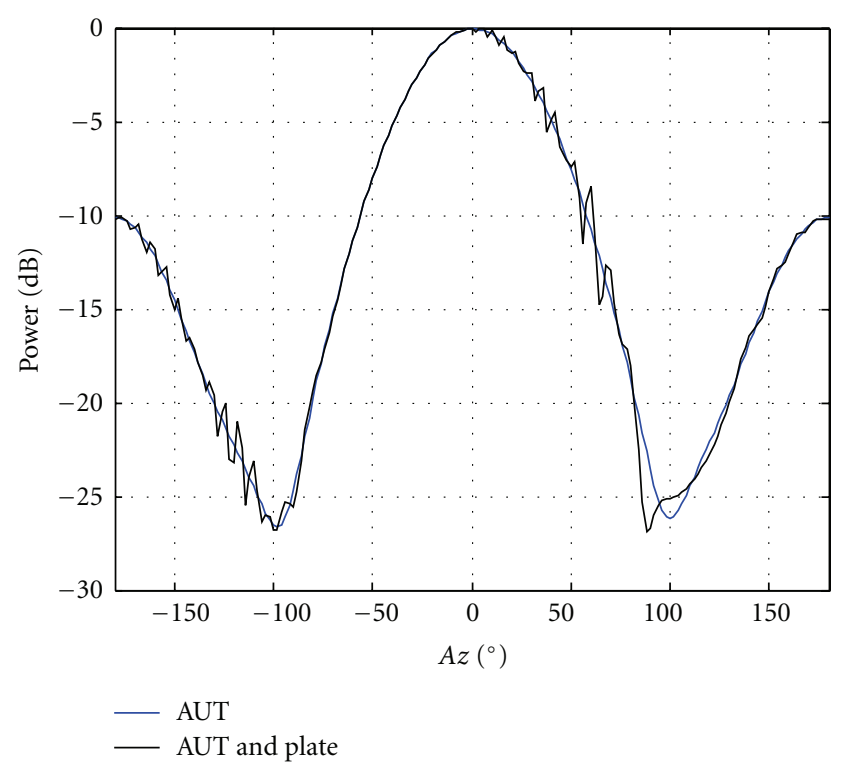

FIGURE 4: Far-field amplitude pattern of OEWG with and without plate. AUT offset $=30 \mathrm{~cm}$.

scatting on the measurements. However, as modelled above, the MARS measurement technique deliberately displaces the AUT away from the centre of rotation. This has the effect of making the differences in the illuminating field far more pronounced than would otherwise be the case, and it is this greater differentiation that makes the identification of scattered fields and their subsequent removal viable. In order that this effect could be further investigated, the simulation was repeated with an AUT offset of $0.3 \mathrm{~m}$ and $0.5 \mathrm{~m}$. The results of these simulations can be found presented in Figures 4 and 5 and Figures 6 and 7, respectively. It is well known that as an antenna is moved away from the centre of rotation 


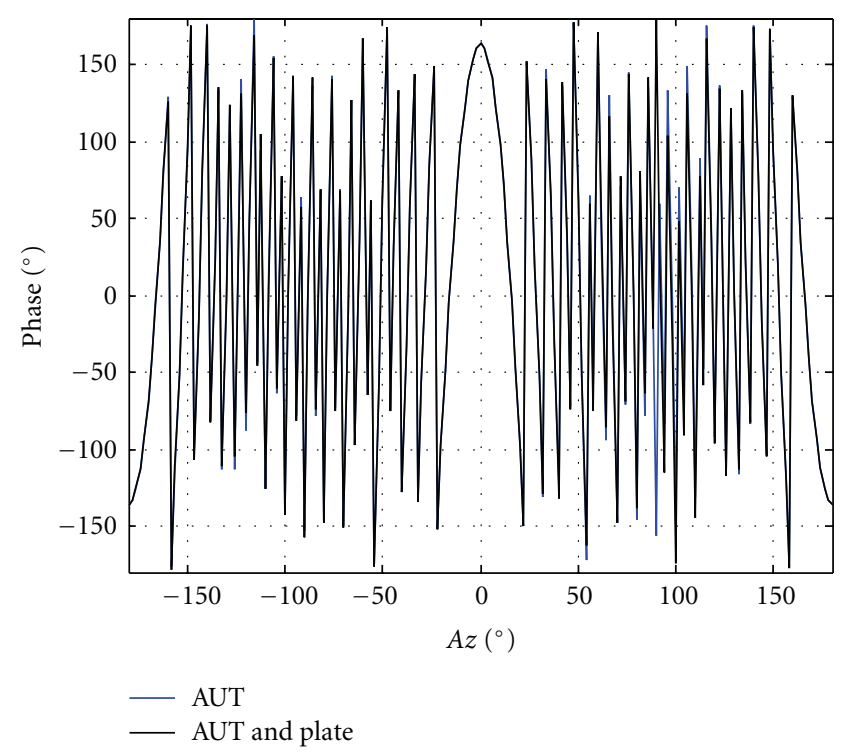

FIGURE 5: Far-field phase pattern of OEWG with and without plate. AUT offset $=30 \mathrm{~cm}$.

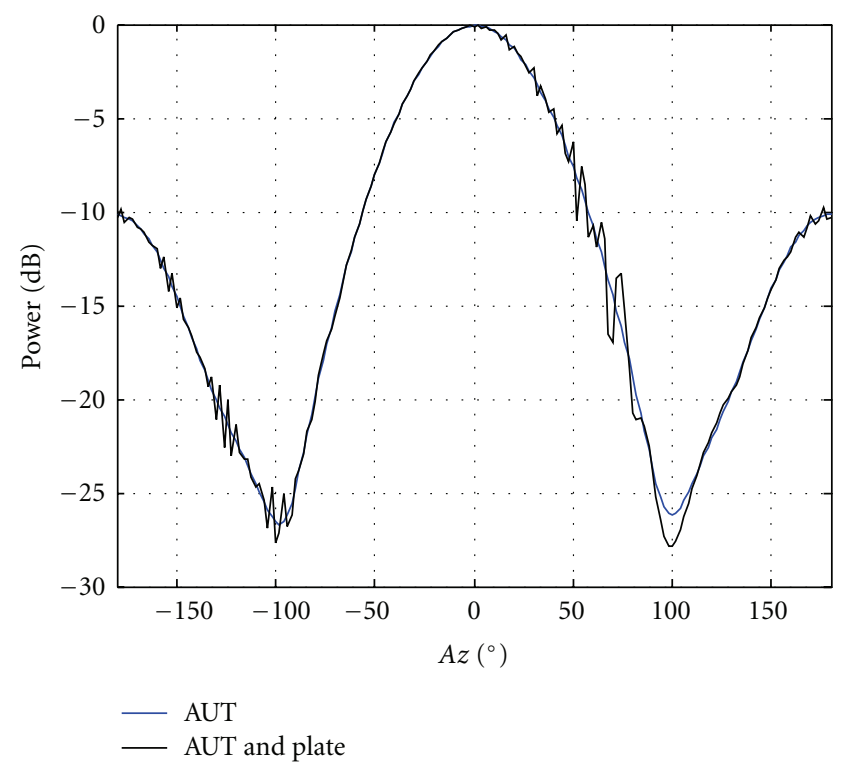

FIGURE 6: Far-field amplitude pattern of OEWG with and without plate. AUT offset $=50 \mathrm{~cm}$.

of the measurement system, in the true far field, the phase function of the AUT changes such that [17]

$$
\underline{E}_{t}(r \longrightarrow \infty, \theta, \phi)=\underline{E}(r \longrightarrow \infty, \theta, \phi) e^{j \underline{k}_{0} \cdot \underline{r}} .
$$

Here, $\underline{r}$ denotes the displacement vector between the centre of the measurement coordinate system and the centre of the current sources, for example, the aperture of the AUT. If a displacement is made purely in the AUT $z$-axis, this reduces to

$$
\underline{E}_{t}(r \longrightarrow \infty, \theta, \phi)=\underline{E}(r \longrightarrow \infty, \theta, \phi) e^{j k_{0} \cos \theta \Delta z} .
$$

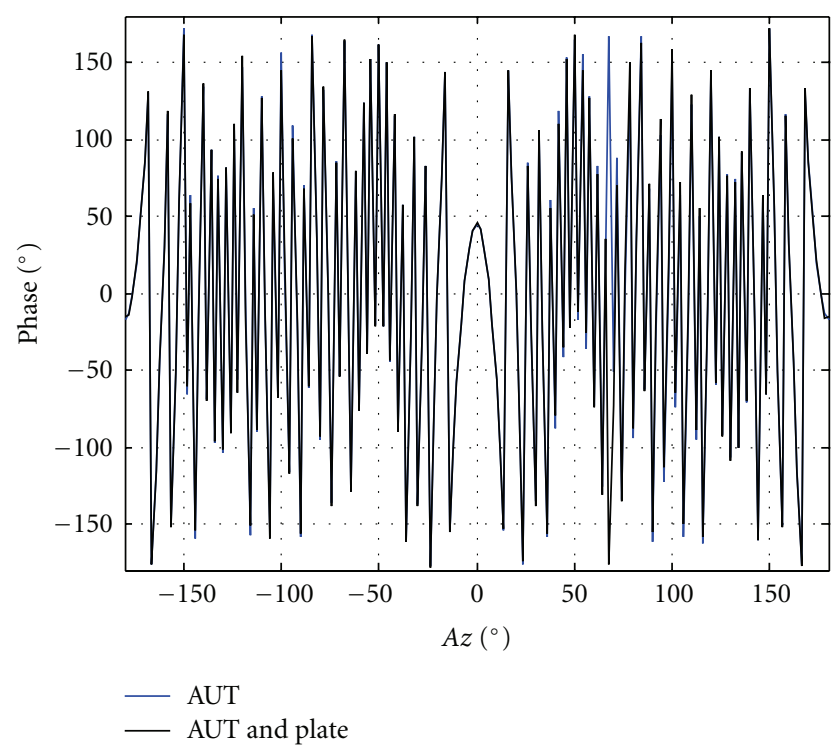

FIGURE 7: Far-field phase pattern of OEWG with and without plate. AUT offset $=50 \mathrm{~cm}$.

Here $\Delta z$ was $0.1,0.3$, and $0.5 \mathrm{~m}$, respectively for the three simulations presented above. As can be seen from the inspection of Figures 3, 5, and, 7 it is clear that a parabolic phase function has been introduced onto the farfield patterns. This feature was not introduced explicitly within the simulation and is an artefact of the geometry of the simulation and the due regard to the phase, that is, inherent within the Kirchhoff-Huygens formula.

The effects of the reflecting plate can be clearly seen on the far-field great circle cuts with disturbances evident primarily around the $0^{\circ}<\theta<90^{\circ}$ angular region with some scattering also being evident on the back lobes of the antenna. This ripple is a result of the direct and indirect (scattered) signals adding in and out of phase as the AUT is progressively rotated. Clearly, the further the AUT is displaced across the range quiet zone during the measurement simulation the more rapidly the signals will beat in and out of phase as the difference in the electrical paths becomes larger and the higher the angular frequency of the resulting ripple will be observed on the measured antenna pattern. Again, this is in agreement with what is generally observed when taking an F-MARS measurement, compare [15, Figures 2, 4, and 6].

It is well known that the electromagnetic fields outside an arbitrary test antenna radiating into free space can be expanded into a set of orthogonal cylindrical mode coefficients (CMC) and that these modes and coefficients can then be used to obtain the electric and magnetic fields everywhere in space outside of a conceptual cylindrical surface which encloses the radiator, that is, which encloses the majority of the current sources [7-9, 14, 15]. The success of F-MARS processing is predicated upon the ability to deduce these CMCs and, on the characteristics of the distribution of those modes once they have been obtained. Thus, in order that this could be investigated in detail, farfield antenna pattern data was simulated for the AUT with 
the aperture located at several different offsets from the origin of the measurement coordinate system to illustrate the effect of the offset on the equivalent CMC, and thus on the MARS correction itself. Figures 8, 10, and 12 show the ideal modelled far-field patterns (red trace) plotted together with the scattering contaminated far-field equivalent plots (blue trace) and the F-MARS processed pattern (black trace) for the cases where the AUT was displaced by $10 \mathrm{~cm}, 30 \mathrm{~cm}$, and $50 \mathrm{~cm}$. Here, it is clear that as the displacement becomes larger, the agreement between the ideal pattern and the F-MARS processed pattern is in increasingly encouraging agreement. Figures 9, 11, and 13 contain the equivalent $\mathrm{CMC}$ plots with (blue trace) and without (red trace) MARS filtering. It is clear from the inspection of these plots that the displacement of the spectral peak of the scattered fields to increasingly higher order modes with increasing AUT offset corresponds to the change in the appearance of the measured far-field patterns, compare [15]. Note that as the offset is increased, the spectral peak representing multipath energy tends to move further away (towards higher order mode numbers) from the AUT spectral peak (shown in the centre of the plot at $n=0$ ). In addition, note that the multipath spectrum becomes wider as the offset is increased. It can be seen that in the small $10 \mathrm{~cm}$ offset case, the multipath and AUT modes are mostly coincident, and thus MARS processing provides less immunity to range multipath. In the case where the offset is larger, that is, $30 \mathrm{~cm}$ and $50 \mathrm{~cm}$ it is clear that the MARS provides far great immunity from multipath as the CMCs that are associated with field reflected from the reflecting plate can now be clearly resolved from the antenna coefficients, which are closely distributed about the lowest order mode. Here, MARS processing provides a clear view of the AUT pattern throughout the range of angles where range multipath had the greatest effect. These observations are in agreement with what has been noted while taking actual range measurements [15].

The minimum conceptual maximum radial extent (MRE) which is used by the MARS cylindrical mode coefficient filter function to exclude scattering from the measurements must be sufficiently large to create a conceptual cylinder that is coaxial with the rotation axis and which encloses the majority of the current sources. As the AUT was a low gain, OEWG inspection of the near-field simulation showed that significant currents flowed down the exterior surfaces of the waveguide walls. For this reason, this antenna is not a true aperture-type antenna as the excitation current distribution extends in all three Cartesian axes. These currents contribute to the far-field pattern, particularly to the wide angle sidelobes, and therefore should not be excluded from the measurement by the F-MARS processing. Thus, during this processing a conservative radius of $8 \mathrm{~cm}$ was employed (which was far larger than suggested by the maximum diagonal dimension of the aperture, which was $2.5 \mathrm{~cm}$ ) as this retained many more of these exterior fields. Thus far, only rectangular brick-wall band-pass filter functions have been used to filter the CMCs when applying far-field MARS. As the exclusion of modes is based upon a consideration of the physical (and therefore electrical) extent of the source, this technique will preserve the integrity of the

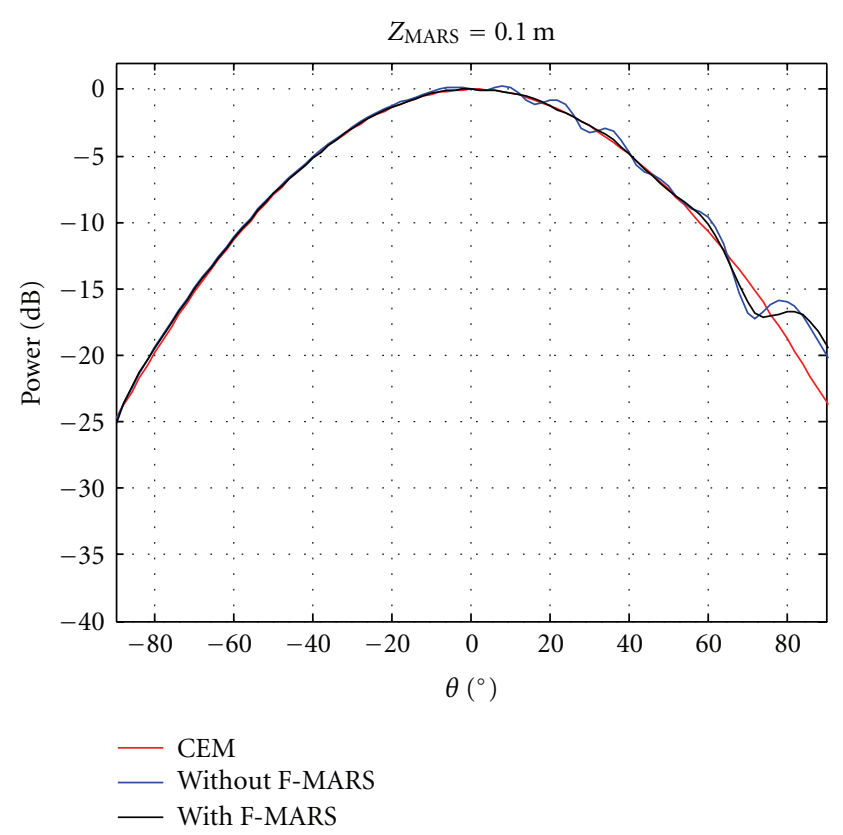

Figure 8: Far-field cut with and without MARS processing. Displacement of $10 \mathrm{~cm}$.

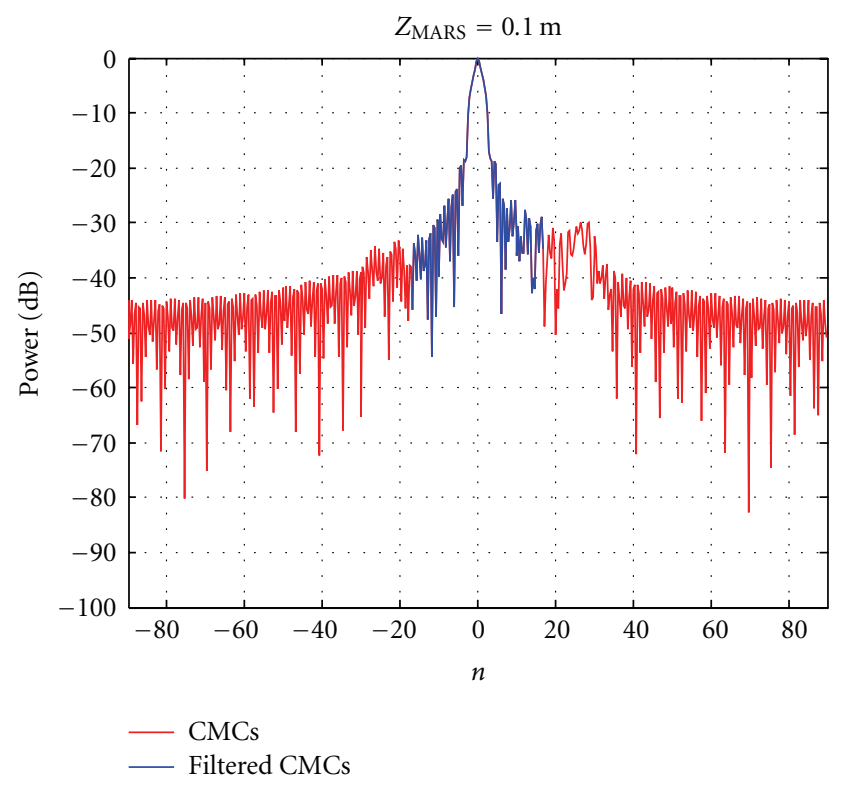

FIgUre 9: Equivalent CMCs with AUT displacement of $10 \mathrm{~cm}$.

underlying pattern function. In an attempt to minimise the likelihood of introducing transform leakage, which would result in a spurious ripple being superimposed on the FMARS filtered far-field pattern, since this is a second-order effect, and as the ideal antenna pattern was known a priori from the CEM simulation for the first time, these effects could be assessed critically.

As there are almost infinite number of possible windowing functions that could be examined, it was decided that merely establishing the effectiveness that this approach has in enabling a tighter band-pass filter functions to be employed 


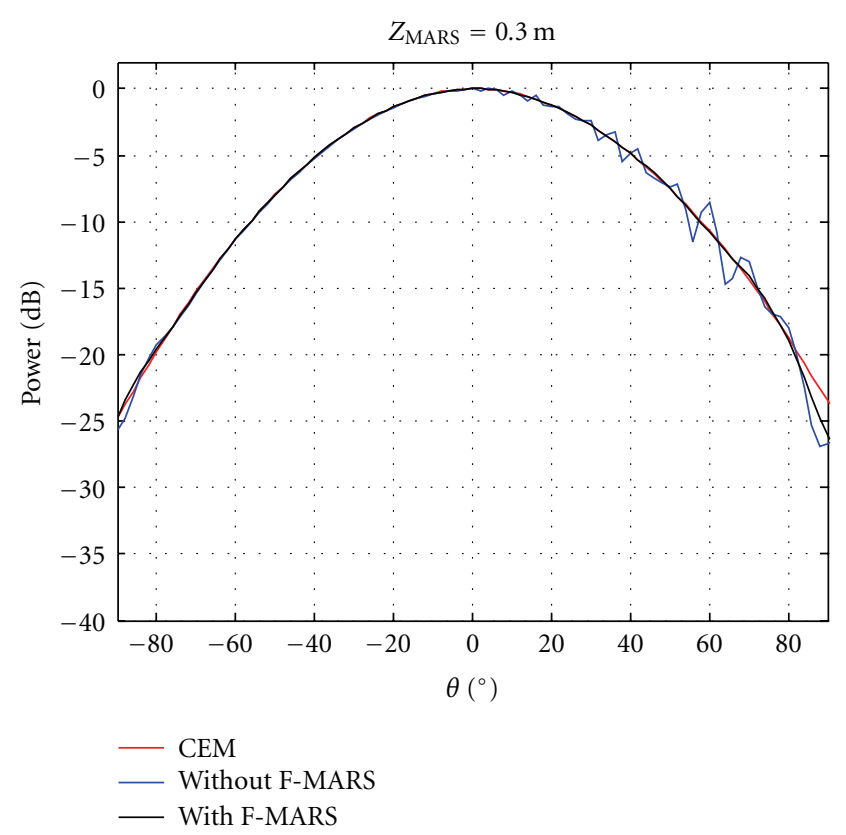

FIGURe 10: Far-field cut with and without MARS processing. Displacement of $30 \mathrm{~cm}$.

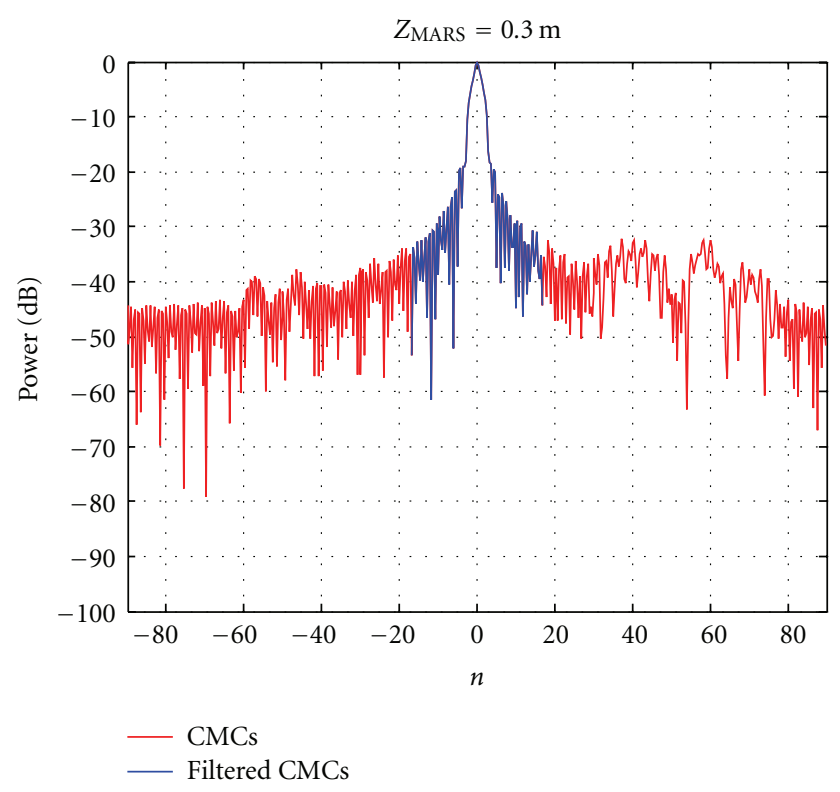

FIgUre 11: Equivalent CMCs with AUT displacement of $30 \mathrm{~cm}$.

was thought sufficient to justify a more exhaustive investigation at a future time. Figure 14 contains an equivalent CMC plot to that shown in Figure 11, only here, a cosine squared windowing function has been applied to filter out higher order modes whilst attempting to match the function and as many of its derivatives to zero at the boundary of the transform domain so as to minimise ripple in the transformed domain. As can be seen from the inspection of Figure 15, the ideal CEM pattern and the F-MARS filtered pattern are in very encouraging agreement. Furthermore, the degree of agreement is clearly an improvement over the

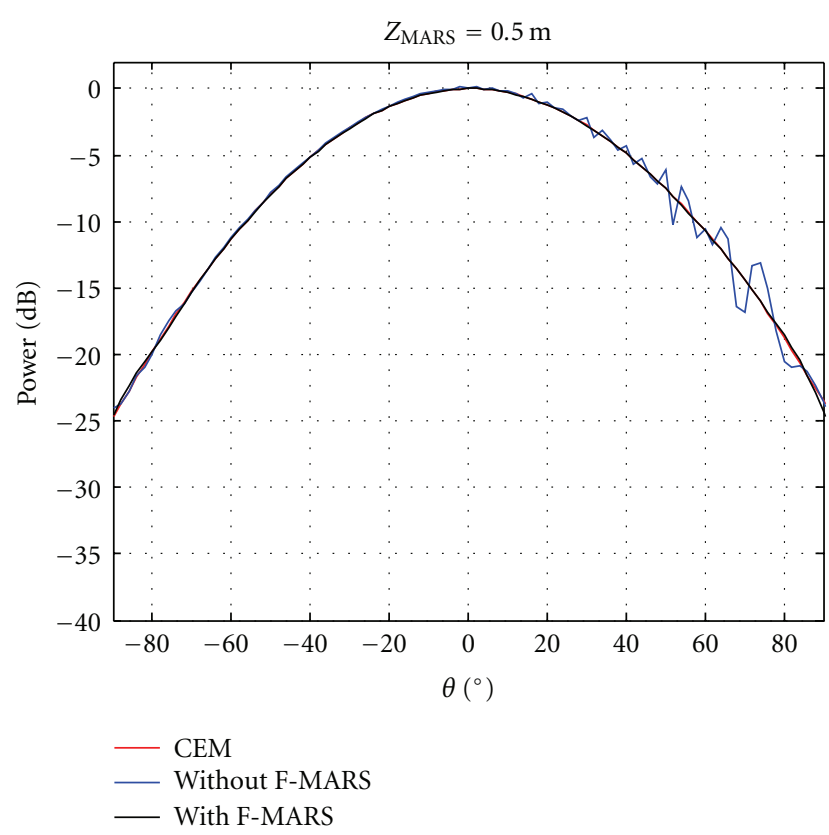

FIGURE 12: Far-field cut with and without MARS processing. Displacement of $50 \mathrm{~cm}$.

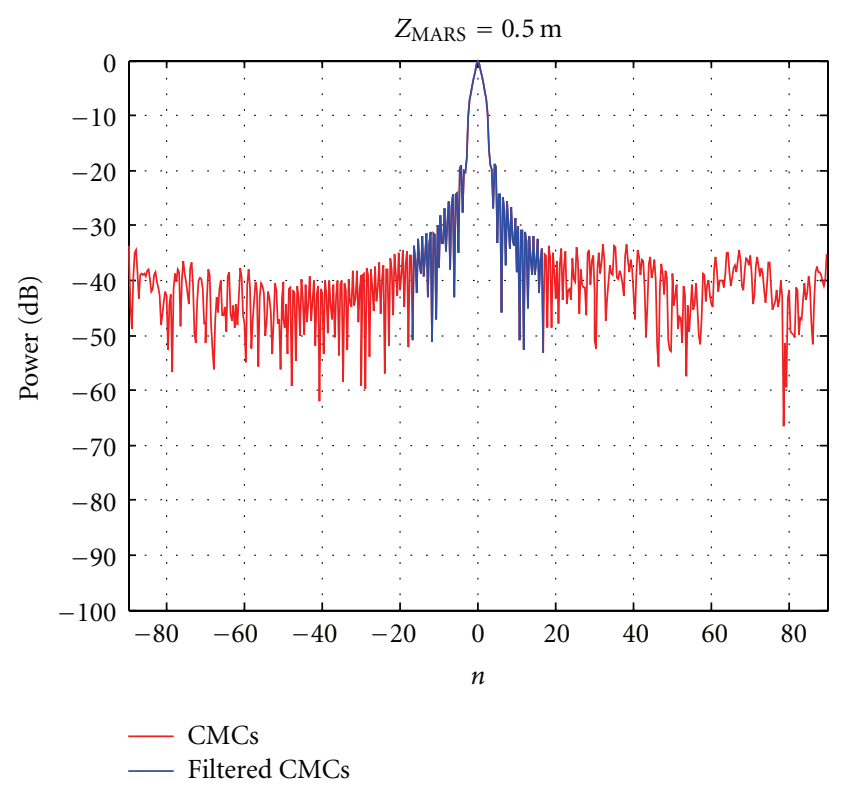

Figure 13: Equivalent CMCs with AUT displacement of $50 \mathrm{~cm}$.

previous case, compare Figure 10, where a rectangular filter function had been employed. This demonstrates the validity of this future line of research.

\section{Summary and Conclusions}

A new physical-optics-based antenna measurement model that can be used to investigate the impact of various error terms within the facility level error budget of a given spherical near- or far-field range has been introduced. A traditional objection to the implementation of physicaloptics-based software modelling tools has been the long 


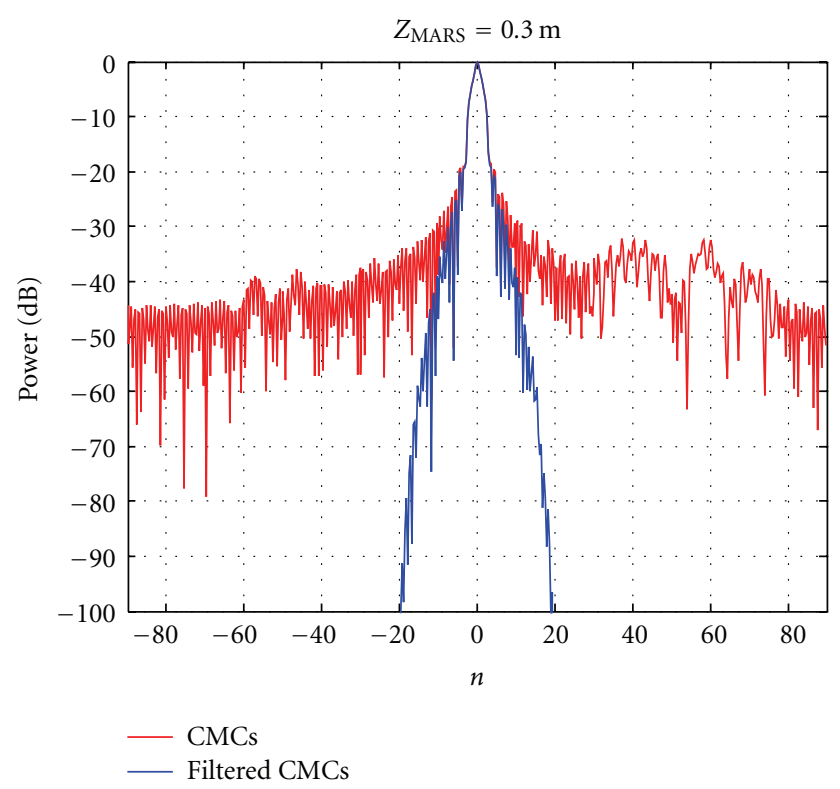

Figure 14: Equivalent CMCs with AUT displacement of $30 \mathrm{~cm}$. Cosine squared filter applied.

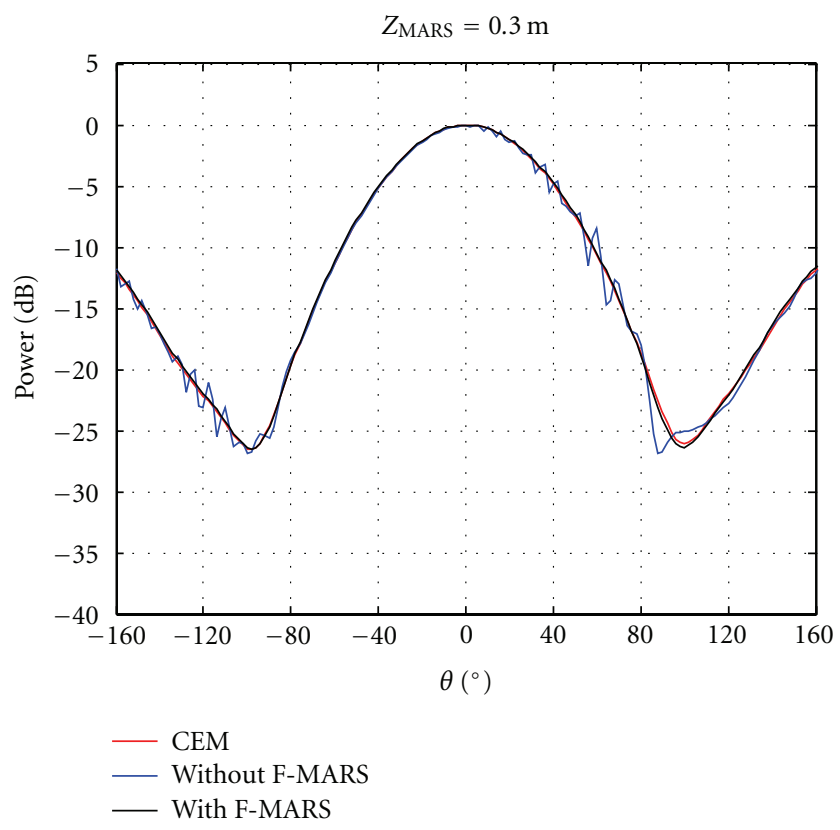

FIGURE 15: Far-field cut with and without MARS processing. Displacement of $30 \mathrm{~cm}$.

run times; however, with the advent of high-CPU-power computers this objection has been largely nullified. This tool has been used to model a conventional F-MARS measurement whereupon similar phenomena have been observed in the CEM model as seen in actual range measurements. Specifically these are as follows: (1) parabolic phase function is imposed on far-field phase pattern that is dependent upon displacement of AUT from measurement origin, (2) the effects of scattering on a far-field pattern depend upon the AUT displacement with greater displacement resulting in higher angular frequency ripple on the far-field pattern, (3) CMCs resulting from scattering are displaced to higher order modes AUT modes are displaced to lower order modes once the AUT is mathematically displaced back to the origin of the measurement coordinate system, (4) the amount of separation between mode distributions associated with scattering and those associated with the AUT increases as the displacement increases, and (5) F-MARS is capable of effectively suppressing scattering providing that the magnitude of the displacement is sufficiently large.

Thus, the CEM model has been able to provide further confirmation of the effectiveness of the far-field MARS technique, and hence F-MARS processing can be used with a very high degree of confidence since all the steps in the measurement and analysis are consistent with the wellestablished principles of the standard cylindrical near-field theory and measurement technique, and all comparisons to date have proved to be overwhelmingly positive. The offset of the AUT and the resulting smaller data point spacing are valid if the data point spacing satisfies the sampling criteria. The translation of the far-field pattern to the origin with the application of a differential phase change is rigorous. The selection of the mode cutoff for the translated pattern is based on the physical dimensions of the AUT and its translated location.

\section{Acknowledgment}

The authors extend their gratitude to the reviewers for their very detailed and constructive comments and suggestions.

\section{References}

[1] A. C. Newell, "Error analysis techniques for planar nearfield measurements." IEEE Transactions on Antennas and Propagation, vol. 36, no. 6, pp. 754-768, 1988.

[2] G. E. Evans, Antenna Measurement Techniques, Artech House, 1990.

[3] G.E. Hindman and A.C. Newell, "Spherical near-field selfcomparison measurements," in 26th AMTA Annual Meeting \& Symposium, Atlanta, Ga, USA, October 2004.

[4] G. E. Hindman and A. C. Newell, "Reflection Suppression in a large spherical near-field range," in 27th AMTA Annual Meeting \& Symposium, Newport, RI, USA, October 2005.

[5] G. E. Hindman and A. C. Newell, "Reflection suppression to improve anechoic chamber performance," in AMTA Europe, Munich, Germany, March 2006.

[6] D. W. Hess, "The IsoFilterTM technique: extension to transverse offsets," in AMTA Symposium, Austin, Tex, USA, 2006.

[7] S. F. Gregson, A. C. Newell, and G. E. Hindman, "Reflection suppression in cylindrical near-field antenna measurement systems - cylindrical MARS," in 31st AMTA Annual Meeting ¿ Symposium, Salt Lake City, Utah, USA, November 2009.

[8] S. F. Gregson, A. C. Newell, and G. E. Hindman, "Reflection suppression in cylindrical near-field measurements of electrically small antennas," in Loughborough Antennas and Propagation Conference (LAPC '09), pp. 393-396, November 2009.

[9] S. F. Gregson, A. C. Newell, G. E. Hindman, and M. J. Carey, "Advances in cylindrical Mathematical Absorber Reflection 
Suppression," in 4th European Conference on Antennas and Propagation (EuCAP'10), April 2010.

[10] S. F. Gregson, A. C. Newell, G. E. Hindman, and M. J. Carey, Extension of the Mathematical Absorber Reflection Suppression Technique to the Planar Near-Field Geometry, AMTA, Atlanta, Ga, USA, 2010.

[11] S. F. Gregson, A. C. Newell, G. E. Hindman, and M. J. Carey, "APplication of Mathematical Absorber Reflection suppression to planar near-field antenna measurements," in 5th European Conference on Antennas and Propagation (EUCAP '11), pp. 3412-3416, April 2011.

[12] S. F. Gregson, A. C. Newell, and G. E. Hindman, Advances in Planar Mathematical Absorber Reflection Suppression, AMTA, Denver, Colo, USA, 2011.

[13] O. M. Bucci, G. D'Elia, and M. D. Migliore, "A general and effective clutter filtering strategy in near-field antenna measurements," IEE Proceedings: Microwaves, Antennas and Propagation, vol. 151, no. 3, pp. 227-235, 2004.

[14] S. F. Gregson, J. Dupuy, C. G. Parini, A.C. Newell, and G. E. Hindman, "Application of mathematical absorber reflection suppression to far-field antenna testing," in Loughborough Antennas \& Propagation Conference (LAPC '11), Loughborough, UK, November 2011.

[15] S. F. Gregson, B. M. Williams, G. F. Masters, A. C. Newell, and G. E. Hindman, Application of Mathematical Absorber Reflection Suppression to Direct Far-Field Antenna Range Measurements, AMTA, Denver, Colo, USA, 2011.

[16] S. Silver, Microwave Antenna Theory and Design, McGraw, New York, NY, USA, 1st edition, 1947.

[17] S. F. Gregson, J. McCormick, and C. G. Parini, Principles of Planar Near-Field Antenna Measurements, The Institution of Engineering and Technology, UK, 2007.

[18] R. H. Clarke and J. Brown, Diffraction Theory and Antennas, Ellis Horwood, 1980.

[19] G. F. Masters and S. F. Gregson, "Coordinate system plotting for antenna measurements," in AMTA Annual Meeting \& Symposium, St. Louis, Mo, USA, 2007.

[20] J. E. Hansen, Ed., Spherical Near-Field Antenna Measurements, IEE, UK, 1988. 

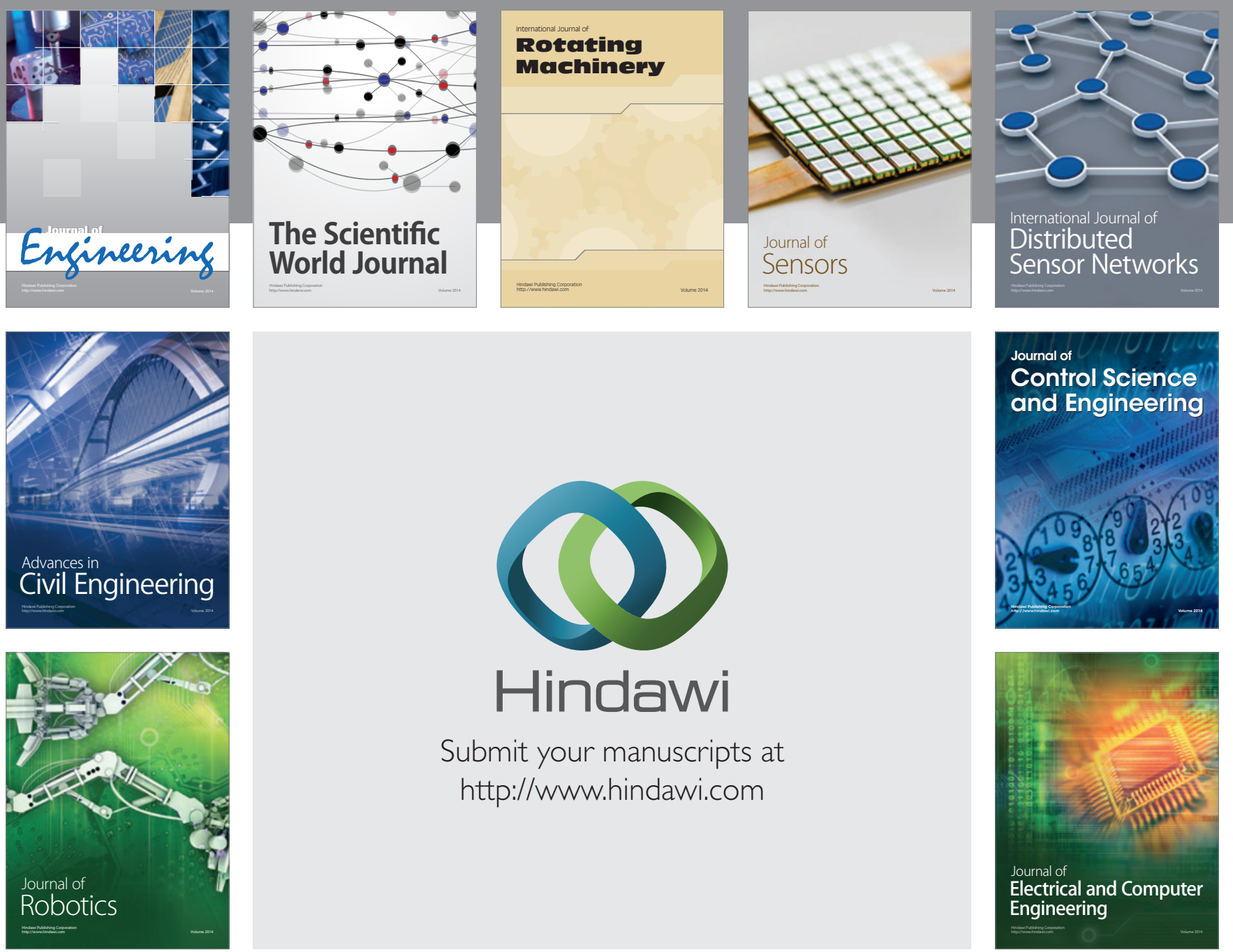

Submit your manuscripts at

http://www.hindawi.com
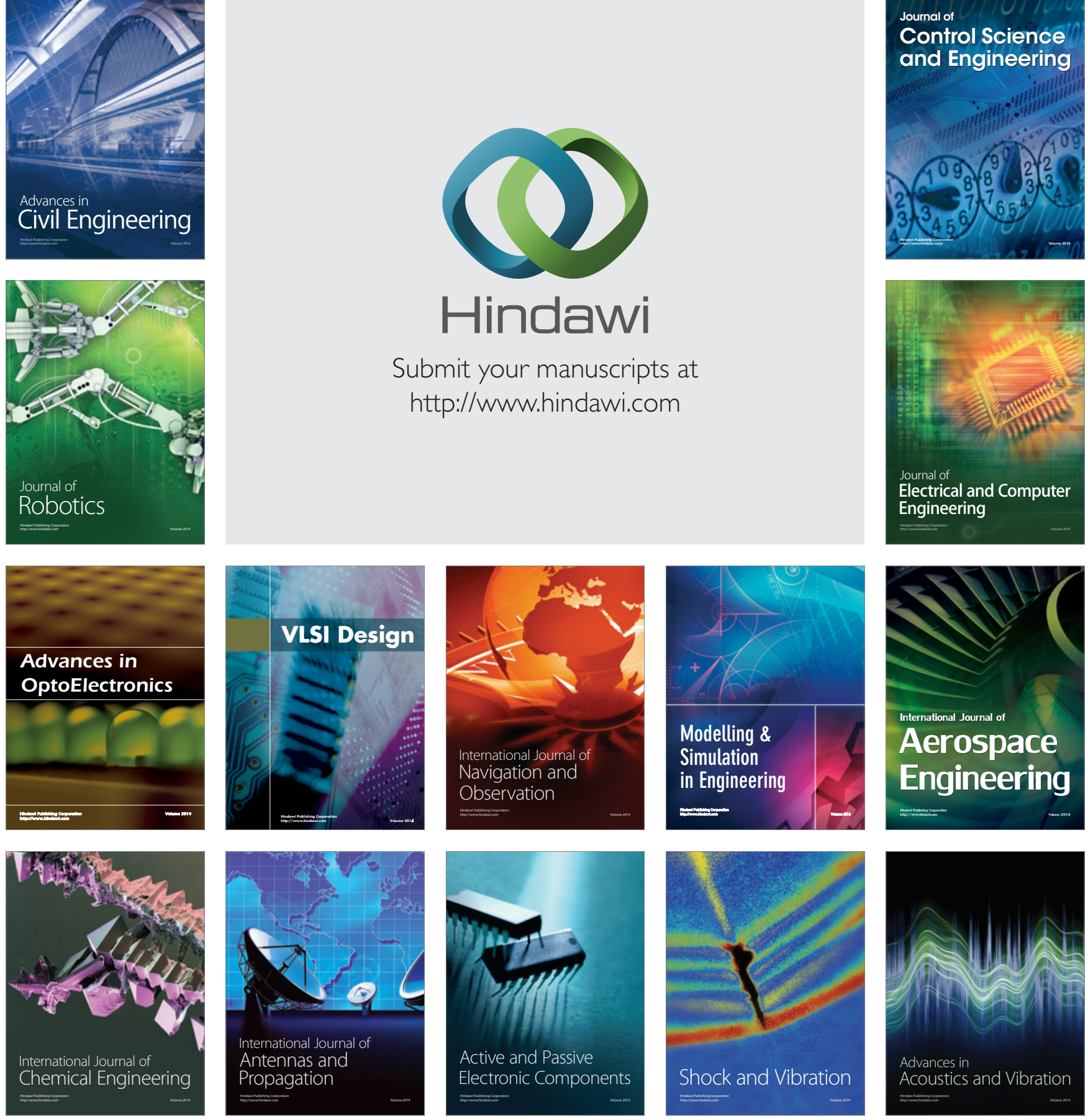